\title{
SAÚDE AUDITIVA, O VÍRUS DA IMUNODEFICIÊNCIA HUMANA E A SÍNDROME DA IMUNODEFICIÊNCIA ADQUIRIDA: UMA REVISÃO
}

\author{
Hearing health, the human immunodeficiency virus and the \\ acquired immunodeficiency syndrome: a review
}

Thais Catalani Morata ${ }^{(1)}$, Maria Cecília Bevilaqua ${ }^{(2)}$, Bianca Simone Zeigelboim ${ }^{(3)}$

\section{RESUMO}

Tema: a Síndrome da Imunodeficiência Adquirida (SIDA/AIDS) é causada pelo Vírus da Imunodeficiência Humana (VIH/HIV), e resulta numa imunidade reduzida, o que torna o indivíduo mais susceptível a doenças e infecções oportunistas. Com o avanço da doença as estruturas do sistema auditivo central podem ser comprometidas pela ação direta do vírus ou decorrente de infecções secundárias e neoplasias. O portador do HIV/AIDS pode também se tornar mais vulnerável a outras patologias do ouvido. Objetivo: o objetivo desta revisão foi exploratório, visando identificar os possíveis pontos de interseção entre distúrbios auditivos e a Síndrome da Imunodeficiência Adquirida. Realizou-se uma revisão da literatura sobre a saúde auditiva dos portadores de HIV/AIDS e foi discutido o impacto potencial de patologias auditivas na qualidade de vida. Conclusão: a literatura sugere que várias possiveis associações existam entre os distúrbios auditivos e a Síndrome da Imunodeficiência Adquirida e o Vírus da Imunodeficiência Humana. Profissionais de saúde, inclusive aqueles dos serviços públicos no Brasil, deveriam examinar a necessidade de iniciativas de saúde auditiva dirigida aos portadores de HIV/AIDS para prevenir patologias auditivas ou reduzir seu impacto na qualidade de vida.

DESCRITORES: Infecções Oportunistas Relacionadas com a AIDS / complicações; Perda auditiva; Risco; Assistência Integral à Saúde; Fatores Socioeconômicos

(1) Fonoaudióloga; Division of Applied Research and Technology National Institute for Occupational Safety and Heath, NIOSH, Cincinnati, EUA; Professora Colaboradora do Programa de Mestrado e Doutorado em Distúrbios da Comunicação da Universidade Tuiuti do Paraná, UTP, Curitiba, PR; Doutora em Ciências e Distúrbios da Comunicação pela Universidade de Cincinnati, EUA, Pós-doutorado em Saúde Ocupacional pela National Academy of Sciences, EUA.

(2) Fonoaudióloga; Coordenadora do Centro de Pesquisas Audiológicas e Professora Titular de Audiologia da Universidade de São Paulo, USP, Bauru, SP; Doutora em Psicologia da Educação pela Pontifícia Universidade Católica de São Paulo, SP; Livre Docência em Audiologia pela Universidade de São Paulo, SP.

(3) Fonoaudióloga; Coordenadora do Programa de Mestrado e Doutorado em Distúrbios da Comunicação da Universidade Tuiuti do Paraná, UTP, Curitiba, PR; Pós-doutorado em Distúrbios da Comunicação Humana pela Universidade Federal de São Paulo/ Escola Paulista de Medicina.

Conflito de interesses: inexistente

\section{INTRODUÇÃO}

Segundo a Organização Mundial de Saúde (OMS), o Brasil tem uma taxa de prevalência de infecção pelo Human Immunodeficiency Vírus (HIV) de $0,6 \%$ concentrada na população de 15 a 49 anos ${ }^{1,2}$. De acordo com o Ministério da Saúde, no Brasil de 1980 a junho de 2007, foram notificados 474.273 casos de Acquired Immune Deficiency Syndrome (AIDS) no País - 289.074 na região Sudeste, 89.250 na região Sul, 53.089 na região Nordeste, 26.757 na região Centro Oeste e 16.103 na região Norte ${ }^{1}$.

O boletim epidemiológico lançado pelo Ministério da Saúde em 2007 revelou, pela primeira vez, dados sobre a proporção de indivíduos que continuaram vivendo com AIDS em até 5 anos após seu diagnóstico. Os dados apontaram que na região Sudeste $90 \%$ dos indivíduos permaneciam vivos. 
Nas outras regiões, os percentuais foram de $78 \%$ na região Norte, $80 \%$ na região Centro Oeste, $81 \%$ na região Nordeste e $82 \%$ na região Sul ${ }^{1}$. Foram registrados 314.294 casos de AIDS em homens e 159.793 em mulheres no período de 1980 a 2006. Ressalta-se, que ao longo do tempo, a diferença na prevalênvia de AIDS entre os sexos vem diminuindo progressivamente. Em 1985, havia 15 casos da doença em homens para 1 mulher. Hoje, a relação é de 1,5 para 1. Em ambos os sexos, a maior parte dos casos se concentra na faixa etária de 25 a 49 anos, porém nos últimos anos, tem-se verificado um aumento percentual de casos na população acima de 50 anos em ambos os sexos ${ }^{1}$.

Em 2005, foram identificados 700 casos de AIDS na população de menores de 5 anos, representando taxa de 3,9 casos por 1000 mil habitantes. Em 2006, foram registrados 526 casos. Considerando as regiões, a taxa de incidência é maior no Sul $(6,1 \%)$, seguido do Sudeste $(4,4 \%)$, Nordeste $(3,1 \%)$, Norte $(2,7 \%)$ e Centro Oeste $(2,6 \%){ }^{1}$

\section{AIDS, HIV e a Pobreza}

O Programa das Nações Unidas para o Desenvolvimento reconhece a associação entre pobreza e a AIDS e postula que a redução da incidência da pobreza absoluta é etapa fundamental no combate desta epidemia fatal ${ }^{3}$. Na verdade, a situação atual da epidemia de HIV, que se torna muito clara nos países em desenvolvimento, indica a existência de um círculo vicioso, aonde a epidemia constitui um grande obstáculo ao desenvolvimento. A doença é debilitante e os doentes têm sua capacidade de trabalho reduzida, o que complica a situação econômica e consequentemente a situação nutricional dos demais membros da família. Quando o doente é o único provedor de sustento familiar a situação se torna muito grave. A doença dá origem a uma série de despesas, na aquisição de medicamentos, de alimentos que habitualmente não são consumidos, no pagamento de despesas de saúde, entre outros. Os pobres ficam mais pobres e com maior vulnerabilidade nutricional. A infecção pelo HIV tem algumas consequências idênticas às da desnutrição crônica: fraca capacidade produtiva, maior susceptibilidade de adoecimento, e absenteísmo. Todos estes fatores agravam a situação provocada pela desnutrição crônica, pois também vai gerar baixa da produtividade ao nível de todo o país, o que vai agravar a pobreza. Esse quadro atinge a saúde geral do indíviduo, o que pode se manifestar de diferentes maneiras.

A pandemia do HIV/AIDS atinge grandes proporções principalmente nos países africanos, pois a pobreza absoluta, pode levar tanto mulheres quanto homens, a prostituírem-se para obter alimento para si e para suas famílias, tornandose assim, agentes de multiplicação da doença. A prostituição é considerada um fator de risco para a AIDS ${ }^{3}$. Somado a este quadro, o HIV/AIDS irá gerar um elevado número de órfãos que irá necessitar de, no mínimo, apoio alimentar e nutricional. O estigma e a discriminação associados à AIDS dificultam o processo de reintegração destas crianças em famílias adotivas. Existe uma certa relutância em assumir responsabilidade por uma criança doente, devido a fatores como medo e ignorância acerca dos mecanismos de transmissão da doença, ou receio pelos encargos financeiros ${ }^{3}$.

Para quebrar o círculo vicioso entre pobreza e HIV/AIDS existe clareza que além da necessidade óbvia de programas preventivos específicos à prevenção da AIDS, são fundamentais ações que acompanhem a promoção de saúde, cuidados de saúde e redução do impacto da doença. Vários programas das Nações Unidas e do Governo no Brasil destacam a necessidade de programas de atenção à saúde materna, infantil, redução de condições de saúde e doenças endêmicas que afetam a população e, contribuem ainda mais para o aumento da pobreza ${ }^{2,3}$.

\section{AIDS, HIV e a Audição}

Estudos recentes com portadores de HIV ou AIDS indicam que esta população é especialmente vulnerável a distúrbios auditivos. Estes distúrbios podem chegar a ter um impacto devastador. A deficiência auditiva dificulta ou torna impossível a comunicação oral, o que tem repercussões diretas no âmbito social e familiar. Dificuldades auditivas criam estigma e afetam a auto-imagem, causando a percepção de incapacidade e de dependência, resultando na marginalização do indivíduo. No adulto ela pode repercutir diretamente na situação econômica, dificultando a obtenção de um emprego ou na possibilidade de se manter empregado. Mesmo nos países desenvolvidos com todas suas possibilidades em atendimento de saúde, estimase que pessoas com deficiências auditivas ganham de 50 a $70 \%$ do que ganham pessoas com audição normal ${ }^{4}$. Além disso, sugere-se que problemas auditivos têm um impacto negativo não só na saúde mental como na saúde em geral ${ }^{5}$. Em crianças, problemas auditivos podem impedir o acesso à educação, o que tem óbvias consequências socioeconômicas.

Considerando os impactos potenciais de problemas auditivos no âmbito de países em desenvolvimento afetados pela AIDS em proporções epidêmicas cabe avaliar se portadores do HIV ou AIDS têm necessidades específicas referentes à sua saúde auditiva. 
O objetivo deste estudo foi realizar uma revisão da literatura sobre a relação entre saúde auditiva e ser portador do vírus HIV ou AIDS e levantar possíveis implicações dessa relação para a saúde pública.

\section{MÉTODOS}

Realizou-se uma revisão narrativa da literatura, usando como questão específica da revisão o objetivo especificado acima. A revisão teve um escopo temático amplo, dado o caracter exploratório deste estudo e estado da arte neste tema ainda incipiente. A busca de literatura foi realizada em dezembro de 2007 e repetida em julho de 2008 . Utilizou-se as bases de dados da Literatura da América Latina e do Caribe em Ciências da Saúde (LILACS), da literatura internacional (MEDLINE e PUBMED), e Base de Dados de Enfermagem (BDENF).

Optou-se pela revisão de literatura sem restrição de período e procedeu-se a uma pesquisa estruturada utilizando o formulário básico com os termos paciente, deficiência auditiva, SIDA, AIDS, HIV, HIV soropositividade, e surdo como palavras-chave. A avaliação dos estudos e a decisão da inclusão de estudos foi realizada por dois dos autores deste estudo (TM e MCB).

\section{REVISÃO DA LITERATURA}

Foram encontradas na literatura um total de 36 artigos consoantes com os objetivos do estudo. Os principais argumentos dos trabalhos encontrados serão descritos a seguir. Um artigo pode ter sido incluído em mais de um subtema.

Claramente, um dos subtemas mais estudados nessa grande área de AIDS, HIV e a Audição é a vulnerabilidade à AIDS na população com deficiência auditiva. A população surda é considerada especialmente vulnerável à AIDS uma vez que, indivíduos com deficiências auditivas profundas têm acesso limitado às informações sobre a doença, e muitas vezes baixo nível de escolaridade. Em comparação com os estudos da ocorrência de AIDS em pacientes com uma deficiência auditiva profunda, os estudos da associação entre deficiência auditiva moderada ou severa e HIV/AIDS são menos numerosos, mas já oferecem evidência clara da associação das duas condições ${ }^{6-16}$.

Um segundo subtema abordado envolve a AIDS ou o HIV como fatores de risco para patologias auditivas. Nos Estados Unidos, o National Institutes of Health estima que $75 \%$ dos adultos portadores da AIDS têm algum tipo de distúrbio auditivo, causado pela própria doença, por infecções oportunistas ou por tratamentos com medicações ototóxicas 17-22.
A alteração auditiva pode ser também secundária a tumores malignos como o Sarcoma de Kapozi devido ao comprometimento do conduto auditivo externo ${ }^{17,23}$.

Crianças portadoras do HIV também se mostram vulneráveis a deficiências auditivas, de diferentes características. A incidência de otite média, por exemplo, foi dominante neste grupo etário enquanto que problemas auditivos centrais predominaram entre adultos ${ }^{18,20,24}$. Importante ressaltar a ocorrência da meningite em casos de AIDS, infecção oportunista comum entre os indivíduos com AIDS ${ }^{25,26}$ que pode levar a um quadro de deficiência auditiva neurossensorial profunda bilateral. No tratamento de pacientes aidéticos ou portadores do HIV, pode ocorrer um problema auditivo pela utilização de drogas anti-retrovirais e/ou medicamentos com potencial ototóxico ${ }^{27-29}$.

Um terceiro subtema aborda especificamente o tipo de patologias observadas na população de portadores de AIDS ou HIV e esta intimamente ligado a um quarto subtema encontrado esta a busca de alternativas para avaliação audiológica da população portadora de AIDS ou HIV. Os estudos citados a seguir em geral cobrem estes dois subtemas.

Alguns estudos ${ }^{30-32}$ referem que a prevalência de alteração auditiva em pacientes com HIV/AIDS varia aproximadamente de 20 a $40 \%$, sendo que a perda auditiva pode ser decorrente de alterações de orelha externa, média e/ou interna ${ }^{33,34}$.

Como mencionado acima, dentre as inúmeras infecções que podem acometer indivíduos com AIDS incluem-se as otites, que podem provocar uma perda auditiva periférica temporária, devendo esta ser identificada o mais precocemente possível ${ }^{21}$. Se não for tratada de forma imeditada e eficaz, a otite pode se tornar crônica e causar uma perda auditiva permanente e neurosensorial.

Em decorrência destes comprometimentos auditivos, podem ser encontradas alterações nos testes que avaliam o processamento auditivo central, refletindo dificuldades na atenção, discriminação, reconhecimento e na compreensão da informação auditiva ${ }^{35}$. Sabe-se que indivíduos com AIDS podem apresentar um comprometimento progressivo do sistema nervoso central, incluindo o sistema auditivo central, pela ação direta do vírus sobre as estruturas destes sistemas, levando a presença de anormalidades eletrofisiológicas nos potencias evocados auditivos de tronco encefálico de média e/ou longa latência incluindo o P300 acompanhando a progressão da doença ${ }^{21,29}$.

Mata Castro et al. ${ }^{30}$ estudaram 30 pacientes infectados pelo HIV utilizando a audiometria tonal e testes eletrofisiológicos buscando a relação entre 
problemas auditivos, o estado clínico dos pacientes, o grau de redução linfocitária por meio do número de linfócitos CD4 e o uso de medicação antirretrovírica. Os autores constataram que 40\% dos pacientes referiram hipoacusia na anamnese, $33 \%$ possuíam audiometria alterada e $56 \%$ dos pacientes apresentavam resultados alterados na pesquisa dos potenciais evocados auditivos de tronco encefálico. Os achados mais frequentes foram: perda auditiva neurossensorial nas frequências agudas, aumento da latência das ondas III, $\mathrm{V}$ e do intervalo interpico I-V. Achados similares em termos de características do problema, assim como da porcentagem de pacientes com problemas auditivos centrais já haviam sido publicados anteriormente ${ }^{24}$.

Matas et al. ${ }^{21}$ estudaram 8 indivíduos com AIDS e, observaram uma alteração na avaliação do potencial cognitivo sugerindo, desta forma, comprometimento da via auditiva em regiões corticais e déficit no processamento cognitivo das informações auditivas. Num estudo similar, Silva et al. ${ }^{22}$ avaliaram 21 indivíduos com HIV/AIDS e observaram alterações nos parâmetros de amplitude e de latência na avaliação do P300. No complexo N1-P2, verificou-se amplitude reduzida e latência aumentada na onda N1. A queixa de problemas auditivos e a audiometria alterada se mostraram associados ao uso de medicação antirretrovíricos e os achados dos potenciais evocados estavam associados ao diagnóstico de AIDS e ao número de linfócitos CD4, em um estágio mais avançado da doença. Segundo vários autores ${ }^{17,29,30}$ as anormalidades eletrofisiológicas nos potenciais evocados auditivos de tronco encefálico e potencial cognitivo (P300) podem ser encontradas precocemente, antes do aparecimento clínico de sintomas como comprometimento neurológico e déficits cognitivos que fazem parte do complexo demencial da AIDS ${ }^{36}$.

Brivio et al. ${ }^{37}$ descreveram o caso de um menino de 6.5 meses de idade que foi tratado com zidovudine (AZT) por 12 meses, tendo sido avaliado por meio da pesquisa dos potenciais evocados auditivos de tronco encefálico pré e pós-tratamento. As latências interpicos I-V foram reduzidas significativamente no período do estudo, mudança que não poderia ter sido atribuída exclusivamente ao processo maturacional. O acompanhamento de uma criança com AIDS por um período de 2 anos, nas idades de 21, 34 e 43 meses, confirmou a redução das latências das ondas relatada no estudo de Brivio et al. ${ }^{38}$, assim como relatou a ocorrência de uma perda auditiva nas altas freqüências subseqüente ao tratamento de 19 meses ${ }^{38}$.
As investigações iniciais deste tema abordavam uma larga gama de problemas otorrinolaringológicos ou neurológicos que envolveriam também a audição, e foi levantado o argumento que doenças otoneurológicas poderiam ser um indicador precoce da existência de uma imunodeficiência ${ }^{39-41}$.

Resumindo, os 36 trabalhos encontrados descritos acima podem ser subdivididos em quatro categorias temáticas: vulnerabilidade à AIDS na população com deficiência auditiva, AIDS/HIV como fatores de risco para patologias auditivas, tipo de patologias observadas na população de portadores de AIDS ou HIV e avaliação audiológica dessa população. Em comparação com o total de publicações sobre portadores de HIV e AIDS, o número de publicações sobre alterações auditivas dessa população é reduzido. Embora o Brasil seja referência mundial em programas de prevenção e tratamento da AIDS, nenhuma ação governamental no Brasil ou em outros países já desenvolveu programas no sentido de monitorar e intervir para promoção da saúde auditiva do paciente portador do HIV ou AIDS.

Para quebrar o círculo vicioso entre pobreza e HIV/AIDS existe clareza em que além da necessidade óbvia de programas preventivos específicos à prevenção da AIDS, são fundamentais ações que acompanhem a promoção e cuidados com a saúde para redução do impacto da doença. Vários programas das Nações Unidas destacam a necessidade de programas de atenção à saúde materna, infantil assim como a redução de doenças endêmicas que afetam a população e contribuem ainda mais para o aumento da pobreza.

Como mencionado anteriormente, o impacto de uma deficiência auditiva pode variar de mínimo até sérío, dependendo do grau do problema, da idade do portador da deficiência e das condições de atendimento de saúde e de estudo ou trabalho existentes na comunidade.

Considerando os impactos potenciais de problemas auditivos descritos acima no âmbito de países em desenvolvimento afetados pela AIDS em proporções epidêmicas, e considerando os fatores apresentados relativos à associação entre a pobreza e a AIDS, torna-se claro que o cuidado com a saúde auditiva da população afetada pelo HIV/AIDS é extremamente relevante. Uma deficiência auditiva poderá maximizar a questão do estigma, isolamento social, dificuldades econômicas, dramaticamente interferindo na qualidade de vida do portador de HIV/AIDS. A necessidade da incorporação de ações de cuidados com a saúde auditiva da população deve ser avaliada como parte das iniciativas de cuidados à saúde e redução do impacto da AIDS. 
Como primeiro passo seria necessária a avaliação da ocorrência e do tipo de distúrbios auditivos da população portadora do vírus da imunodeficiência humana ou de AIDS de diferentes faixas etárias e níveis socioeconômicos. Como passo seguinte, um modelo pioneiro de intervenção precisa ser criado e testado. $O$ desenvolvimento e avaliação de intervenções sustentáveis para a vigilância epidemiológica de populações infectadas pelo HIV em países em desenvolvimento, concernentes à saúde auditiva, poderia ser exportável a outros países com o perfil brasileiro.

\section{CONCLUSÃO}

A literatura sugere que profissionais de saúde deveriam examinar a necessidade de iniciativas de saúde auditiva dirigida aos portadores de HIV/ AIDS para prevenir patologias auditivas ou reduzir seu impacto na qualidade de vida e, de sua possível inserção nos Serviços de Saúde Auditiva de Média/ Alta Complexidade do Sistema SUS. Tal iniciativa culmiraria com a melhoria da qualidade de vida por portadores de HIV/AIDS, através da melhoria dos cuidados à saúde desta população e da redução do impacto da doença.

\begin{abstract}
Background: Acquired Immunodeficiency Syndrome (or Acquired immune deficiency syndrome or AIDS) is caused by the Human Immunodeficiency Virus (HIV) and results in reduced immunity, leaving affected individuals more susceptible to illness and opportunistic infections. As the disease progresses, structures within the central auditory system can be affected either by the direct action of the virus or as a consequence of secondary infections. Other areas of the auditory system may also be more vulnerable to pathology in people living with HIV or AIDS. Purpose: the goal of this review was exploratory, to identify possible points of intersection between auditory disorders and Acquired Immune Deficiency Syndrome. This paper reports the results of a literature review on auditory disorders associated with HIV and AIDS and discusses the potential impact of ear pathologies among HIV/AIDSinfected populations. Conclusion: literature suggests several possible types of association between auditory disorders and the Acquired Immune Deficiency Syndrome and the Human Immunodeficiency Virus, and that people with HIV/AIDS may require hearing care interventions. Health professionals, including those from the Brazilian public health system, should examine the need for hearing-related services among HIV/AIDS patients in order to reduce the impact of the disease on daily life activities and prevent insofar, further auditory deterioration.
\end{abstract}

KEYWORDS: AIDS-Related Opportunistic Infections/ complications; Hearing Loss; Risk; Comprehensive Health Care; Socioeconomic Factors

\section{REFERÊNCIAS}

1. AIDS e o HIV. [homepage na internet] Brasil: Ministério da Saúde. Portal de Serviços do Governo. [atualizada em fevereiro de 2008; acesso em 27 ago 2008]. Disponível em: URL: http://www.aids.gov.br

2. The Joint United Nations Programme on HIV/AIDS. Report on the global AIDS epidemic. [homepage na internet] Genebra: United Nations, UNAIDS. [atualizada em abril de 2007; acesso em 27 ago 2008] Disponível em: URL: http://www.unAIDS.org/ en/HIV_data/2006GlobalReport/default.asp

3. Food and Agriculture Organization of the United Nations/ World Health Organization (FAO/WHO). Feeding hope: nutrition plays key role in HIV/AIDS care. WHO: Roma e Genebra; 25 Feb 2003.
4. Mohr PE, Feldman JJ, Dunbar JL. The societal costs of severe to profound hearing loss in the United States. Int J Technol Assess Health Care. 2000; 16(4):1120-35.

5. Van Oyen H, Tafforeau J, Demarest S. The impact of hearing disability on well-being and health. Soz Praventivmed. 2001; 46(5):335-43.

6. Bat-Chava Y, Martin D, Kosciw JG. Barriers to HIV/AIDS knowledge and prevention among deaf and hard of hearing people. AIDS Care. 2005; 17(5):623-34.

7. Gaskins S. Special population: HIV/AIDS among the deaf and hard of hearing. J Assoc Nurses AIDS Care. 1999; 10(2):75-8.

8. Groce NE, Yousafzai AK, Van der Maas F. HIV/AIDS and disability: differences in HIV/AIDS 
knowledge between deaf and hearing people in Nigeria. Disabil Rehabil. 2007; 15(5):367-71.

9. Mallinson RK. Perceptions of HIV/AIDS by deaf gay men. J Assoc Nurses AIDS Care. 2004; 15(4):27-36.

10. Peinkofer JR. HIV education for the deaf, a vulnerable minority. Public Health Rep. 1994; 109(3):390-6.

11. Groce N, Yousafzai A, Dlamini $P$, Zalud $S$, Wirz S. HIV/AIDS and disability: a pilot survey of HIV/AIDS knowledge among a deaf population in Swaziland. Int J Rehabil Res. 2006; 29(4):319-24. 12. Heuttel KL, Rothstein WG. HIV/AIDS knowledge and information sources among deaf and hearing college students. Am Ann Deaf. 2001; 146(3):280-6. 13. Mallinson RK. Perceptions of HIV/AIDS by deaf gay men. J Assoc Nurses AIDS Care. 2004; 15(4):27-36.

14. Stevens $H$. AIDS, not hearing AIDS: exploring the link between the deaf community and HIV/AIDS. Health Hum Rights. 1998; 2(4):98-113.

15. Van Biema D. AIDS. In one community, silence equals death. Thousands of deaf Americans have never learned the details about HIV and AIDS and their ignorance is killing them. Time. 1994; 143(14):76-7.

16. Woodroffe T, Gorenflo DW, Meador HE, Zazove P. Knowledge and attitudes about AIDS among deaf and hard of hearing persons. AIDS Care. 1998; 10(3):377-86.

17. Birchall MA, Wight RG, French PD, Cockbain $Z$, Smith SJ. Auditory function in patients infected with the human immunodeficiency virus. Clin Otolaryngol. 1992; 17(2):117-21.

18. Bankaitis $A E$. The effects of click rate on the auditory brain stem response (ABR) in patients with varying degrees of HIV-infection: a pilot study. Ear Hear. 1995; 16(3):321- 4.

19. AIDS alert. AIDS patients often have hearing and speech problems. AIDS Alert. 1999; 14(8):1-2.

20. Zuniga J. Communication disorders and HIV disease. J Int Assoc Physicians AIDS Care. 1999; 5(4):16-23.

21. Matas CG, Juan KR, Nakano RA. Potenciais evocados auditivos de media e longa latência em adultos com AIDS. Pró-Fono. 2006; 18(2):171-6.

22. Silva AC, Pinto FR, Matas CG. Potenciais evocados auditivos de longa latência em adultos com HIV/AIDS. Pró-Fono. 2007; 19(4):352-6.

23. Gonçalves DU, Antonio Carlos TJ, Becker CG, Gonçalves TML, Greco DB. Manifestações otorrinolaringológicas em pacientes HIV-soro positivo. Rev Bras Otorrinolaringol. 1994; 60(4):267-70.

24. Rosenhall U, Hakansson C, Lowhagen GB, Hanner P, Jonsson-Ehk B. Otoneurological abnormalities in asymptomatic HIV-seropositive patients. Acta Neurol Scand. 1989; 79(2):140-5.

25. Mohan S, Ahmed SI, Alao OA, Schliep TC. A case of AIDS associated cryptococcal meningitis with multiple cranial nerve neuropathies. Clin Neurol Neurosurg. 2006; 108(6):610-3

26. Molyneux E. Bacterial infections in children with HIV/AIDS. Trop Doct. 2004; 34(4):195-8.

27. Simdom J, Watters D, Barlett S, Connick E. Ototoxicity associated with use of nucleoside analog reverse transcriptase inhibitors: a report of 3 possible cases and review of the literature. Clin Infect Dis. 2000; 34(3):2100-2.

28. Williams B. Ototoxicity may be associated with protease inhibitor therapy. Clin Infect Dis. 2001; 33(12):2100-2.

29. Reyes CL, Silva RA, Ysunza RA, Jimenez RG, Berruecos VP, Romo GG. Brainstem auditory evoked response in HIV: infected patients with and without AIDS. Arch Med Res Mex. 2002; 33(1):25-8. 30. Mata Castro N, Yebra Bango M, Tutor de Ureta P, Villarreal García-Lomas M, García López F. Hipoacusia e infección por el virus de la inmunodeficiencia humana. Estudio de 30 pacientes. Rev Clin Esp. 2000; 200(5):271-4.

31. Chandrasekhar SS, Connely PE, Brahmbhatt SS, Shah CS Kloser PC, Baredes S. Otologic and audiologic evaluation of human immunodeficiency virus-infected patients. Am J Otolaryngol. 2000; 21(1):1-9.

32. Khoza K, Ross E. Auditory function in a group of adults infected with HIV/AIDS in Gauteng, South Africa. S Afr J Commun Disord. 2002; 49:17-27.

33. Madriz JJ, Herrera G. Human immunodeficiency virus and acquired immune deficiency syndrome AIDS - related hearing disorders. J Am Acad Audiol. 1995; 6(5):358-64.

34. Bankaitis AE, Keith RW. Audiological changes associated with HIV infection. Ear Nose Throat J. 1995; 74(5):353-9.

35. Roland Junior JT, Alexiades G, Jackman AH, Hillman D, Shapiro W. Cochlear implantation in human immunodeficiency virus: infected patients. Otol Neurotol. 2003; 24(6):892-5.

36. Ollo C, Johnson R, Grafman J. Signs of cognitive change in HIV disease: an event-related brain potential study. Neurol. 1991; 41:209-15.

37. Brivio L, Tornaghi R, Musetti L, Marchisio P, Principi N. Improvement of auditory brainstem responses after treatment with zidovudine in a child with AIDS. Pediatr Neurol. 1992; 7(1):53-5.

38. Christensen LA, Morehouse CR, Powell TW, Alchediak T, Silio M. Antiviral therapy in a child with pediatric human immunodeficiency virus (HIV): case study of audiologic findings. J Am Acad Audiol. 1998; 9(4):292-8. 
39. Barzan L, Carbone A, Tirelli U, Crosato $I M$, Vaccher E, Volpe $R$, et al. Role of the otorhinolaryngologist in HIV infections (em Italiano). Acta Otorhinolaryngol Ital. 1990; 10(1):3-9.

40. Hadfield PJ, Birchall MA, Novelli V, Bailey CM. The ENT manifestations of HIV infection in children. Clin Otolaryngol Allied Sci. 1996; 21(1):30-6.

41. Sacko HB, Ag Mohamed A, Maiga MY, Kalle A, Doumbia SY, Guindo A. [ORL manifestations observed in AIDS. Apropos of 65 cases] Bull Soc Pathol Exot. 1995 Apr; 88(5):234-5.

DOI: 10.1590/S1516-18462010005000052

RECEBIDO EM: 29/06/2009

ACEITO EM: 11/03/2010

Thais Catalani Morata

National Institute for Occupational Safety and

Heath (NIOSH)

4676 Columbia Parkway Cincinnati, OH 45226

Estados Unidos da América

E-mail: tmorata@cdc.gov 